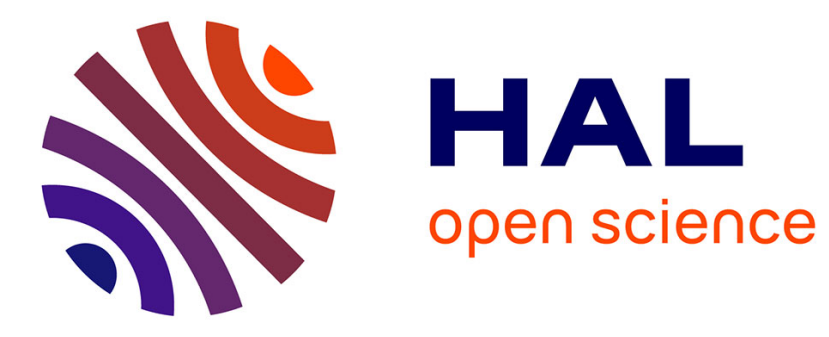

\title{
Interdisciplinary Round-Robin Test on Molecular Spectroscopy of the U(VI) Acetate System
}

Katharina Müller, Harald Foerstendorf, Robin Steudtner, Satoru Tsushima, Michael Kumke, Grégory Lefèvre, Jörg Rothe, Harris Mason, Zoltan Szabo, Ping Yang, et al.

\section{To cite this version:}

Katharina Müller, Harald Foerstendorf, Robin Steudtner, Satoru Tsushima, Michael Kumke, et al.. Interdisciplinary Round-Robin Test on Molecular Spectroscopy of the U(VI) Acetate System. ACS Omega, 2019, 4 (5), pp.8167-8177. 10.1021/acsomega.9b00164 . cea-02120694

\section{HAL Id: cea-02120694 https://hal-cea.archives-ouvertes.fr/cea-02120694}

Submitted on 15 Dec 2020

HAL is a multi-disciplinary open access archive for the deposit and dissemination of scientific research documents, whether they are published or not. The documents may come from teaching and research institutions in France or abroad, or from public or private research centers.
L'archive ouverte pluridisciplinaire HAL, est destinée au dépôt et à la diffusion de documents scientifiques de niveau recherche, publiés ou non, émanant des établissements d'enseignement et de recherche français ou étrangers, des laboratoires publics ou privés. 


\section{Interdisciplinary Round-Robin Test on Molecular Spectroscopy of the U(VI) Acetate System}

Katharina Müller, ${ }^{* \dagger \odot}$ Harald Foerstendorf, ${ }^{\dagger \odot}$ Robin Steudtner, ${ }^{\dagger}$ Satoru Tsushima, ${ }^{\dagger \dagger \odot}$ Michael U. Kumke, ${ }^{\S \odot}$ Grégory Lefêvre, ${ }^{\| \odot}$ Jörg Rothe, ${ }^{\perp}$ Harris Mason, ${ }^{\#}$ Z Zoltán Szabó, ${ }^{\text {II }}$ Ping Yang, ${ }^{\nabla \odot}$ Christian K. R. Adam, ${ }^{\perp \odot}$ Rémi André, Katlen Brennenstuhl, ${ }^{\S}$ Ion Chiorescu, Herman M. Cho, ${ }^{\dagger} \odot$ Gaëlle Creff, ${ }^{\dagger}$ Frédéric Coppin, ${ }^{\S \S}$ Kathy Dardenne, ${ }^{\perp}$ Christophe Den Auwer, ${ }^{\ddagger \star \odot}$ Björn Drobot, ${ }^{\dagger, \| l \mid \odot ~ S a s c h a ~ E i d n e r, ~}{ }^{\S}$ Nancy J. Hess, ${ }^{\perp \perp}$ Peter Kaden, ${ }^{\dagger, \perp \odot}$ Alena Kremleva, ${ }^{\circledR}$ Jerome Kretzschmar, ${ }^{\dagger}$ Sven Krüger, James A. Platts, ${ }^{\# \odot ~ P e t r a ~ J . ~ P a n a k, ~}{ }^{\perp, \text { II }}$ Robert Polly, ${ }^{\perp \odot}$ Brian A. Powell, ${ }^{\nabla \nabla} \odot$ Thomas Rabung, $^{\perp}$ Roland Redon, Pascal E. Reiller, ${ }^{\diamond}$ Notker Rösch, André Rossberg, ${ }^{\dagger,+*}$ Andreas C. Scheinost, ${ }^{\dagger,+* \bullet \odot ~ B e r n d ~ S c h i m m e l p f e n n i g, ~}{ }^{\perp}$ Georg Schreckenbach, ${ }^{\S \S \S \odot ~}$ Andrej Skerencak-Frech, ${ }^{\perp, I I I \odot ~ V l a d i m i r ~ S l a d k o v, ~}\|\| \| \odot$ Pier Lorenzo Solari, ${ }^{\perp \perp \perp}$ Zheming Wang, ${ }^{\perp \perp}$ Nancy M. Washton, ${ }^{\# \#}$ and Xiaobin Zhang ${ }^{\S \S \S}$

${ }^{\dagger}$ Institute of Resource Ecology, Helmholtz-Zentrum Dresden-Rossendorf, Bautzner Landstr. 400, D-01328 Dresden, Germany

${ }^{\ddagger}$ Institute of Innovative Research, Tokyo Tech World Research Hub Initiative (WRHI), Tokyo Institute of Technology, 152-8550 Tokyo, Japan

${ }^{\S}$ Institute of Chemistry, University of Potsdam, 14476 Potsdam, Germany

${ }$ Chimie ParisTech, PSL Research University, CNRS, Institut de Recherche de Chimie Paris (IRCP), F-75005 Paris, France

${ }^{\perp}$ Institute for Nuclear Waste Disposal (KIT-INE), Karlsruhe Institute of Technology, P.O. Box 3640, 76021 Karlsruhe, Germany

\# Physical and Life Science Directorate, Lawrence Livermore National Laboratory, 7000 East Avenue, Livermore, 94550 California, United States

${ }^{\text {II }}$ School of Chemistry, Organic Chemistry, Royal Institute of Technology, S-100 44 Stockholm, Sweden

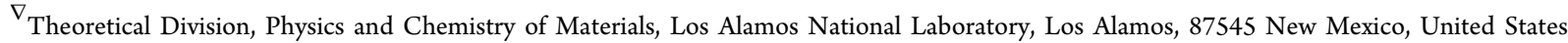

Laboratoire LIS-UMR CNRS 7020, Aix-Marseille Université, Université de Toulon, 83041 Toulon Cedex 9, France

Department of Chemistry, Technische Universität München, Lichtenbergstr. 4, 85748 Garching, Germany

${ }^{\dagger \dagger}$ Physical and Computational Sciences Directorate, Pacific Northwest National Laboratory, MS K2-57, Richland, 99352 Washington, United States

\$tUniversité Côte d'Azur, CNRS, Institut de Chimie de Nice, UMR7272, 06108 Nice, France

${ }^{\S}$ Institut de Radioprotection et de Sûreté Nucléaire (IRSN/PSE-ENV/SRTE/LR2T), CE Cadarache, BP3, 13115 Saint Paul lez Durance, France

"III Central Radionuclide Laboratory, Technische Universität Dresden, Zellescher Weg 19, 01069 Dresden, Germany

${ }^{\perp \perp}$ Environmental Molecular Sciences Laboratory, Pacific Northwest National Laboratory, 3335 Innovation Blvd, Richland, 99354 Washington, United States

\#\# School of Chemistry, Cardiff University, Park Place, CF10 3AT Cardiff, U.K.

IIII Institute of Physical Chemistry, Heidelberg University, Im Neuenheimer Feld 253, D-69120 Heidelberg, Germany

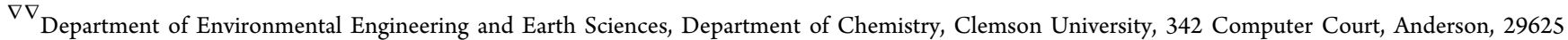
South Carolina, United States

${ }^{\circ}$ Laboratoire MIO-CS 60584, Université de Toulon, 83041 Toulon cedex 9, France

$\checkmark$ Den-Service d'Études Analytiques et de Réactivité des Surfaces (SEARS), CEA, Université Paris-Saclay, F 91191 Gif-sur-Yvette, France

${ }^{\dagger \dagger}$ Institute of High Performance Computing, Agency for Science, Technology and Research, 1 Fusionopolis Way, \#16-16 Connexis, Singapore, Singapore 138632

\$\$ The Rossendorf Beamline (BM20), European Synchrotron Radiation Facility, B.P. 220, F-38043 Grenoble Cedex, France

$\S \S \S$ Department of Chemistry, University of Manitoba, 144 Dysart Road, Winnipeg, Manitoba, R3T 2N2, Canada

||||| Institut de Physique Nucléaire (IPN), CNRS/IN2P3, Université Paris-Sud, 91406 Orsay, France

${ }^{\perp \perp \perp}$ Synchrotron SOLEIL, Ligne de lumière MARS, L'Orme des Merisiers, Saint-Aubin, BP 48, F-91192 Gif-sur-Yvette Cedex, France

\#\#\# Pacific Northwest National Laboratory, 902 Battelle Blvd, Richland, 99352 Washington, United States

Supporting Information

Received: January 17, 2019

Accepted: April 10, 2019

Published: May 3, 2019 
ABSTRACT: A comprehensive molecular analysis of a simple aqueous complexing system-U(VI) acetate-selected to be independently investigated by various spectroscopic (vibrational, luminescence, X-ray absorption, and nuclear magnetic resonance spectroscopy) and quantum chemical methods was achieved by an international round-robin test (RRT). Twenty laboratories from six different countries with a focus on actinide or geochemical research participated and contributed to this scientific endeavor. The outcomes of this RRT were considered on two levels of complexity: first, within each technical discipline, conformities as well as discrepancies of the

Round-Robin Test in Molecular Spectroscopy

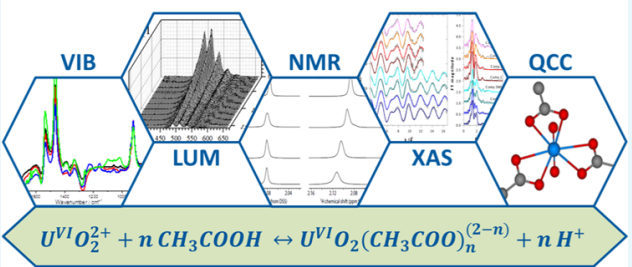
results and their sources were evaluated. The raw data from the different experimental approaches were found to be generally consistent. In particular, for complex setups such as accelerator-based X-ray absorption spectroscopy, the agreement between the raw data was high. By contrast, luminescence spectroscopic data turned out to be strongly related to the chosen acquisition parameters. Second, the potentials and limitations of coupling various spectroscopic and theoretical approaches for the comprehensive study of actinide molecular complexes were assessed. Previous spectroscopic data from the literature were revised and the benchmark data on the $\mathrm{U}(\mathrm{VI})$ acetate system provided an unambiguous molecular interpretation based on the correlation of spectroscopic and theoretical results. The multimethodologic approach and the conclusions drawn address not only important aspects of actinide spectroscopy but particularly general aspects of modern molecular analytical chemistry.

\section{INTRODUCTION}

Exploring the migration pathways of contaminants in the ecosphere is a major field in the environmental sciences. In the past few decades, particular attention was paid to the migration behavior of radioactive heavy metals because of their chemoand radiotoxicity in humans. ${ }^{1}$ The dispersion of these elements in soils and rocks is mainly governed by their chemical speciation in the pore water and accordingly by the chemical reactions occurring at mineral-liquid interfaces. ${ }^{2,3}$ Among the radionuclides, the high abundance of uranium (U) at sites considered for remediation activities and its relevance to the long-term safety assessment of nuclear waste repositories have led to numerous studies on the hydrolysis reactions of the hexavalent, highly soluble uranyl cation $\mathrm{UO}_{2}{ }^{2+}$ and its complexing reactions with ubiquitous inorganic and organic ligands. ${ }^{4-7}$ Additionally, waste forms from other parts of the nuclear fuel cycle are of concern such as remedies of the uranium mining, milling, and enrichment industries, or uranium from other industrial processes, for example geothermal energy generation or phosphate fertilizer production. ${ }^{8}$

Previously, the thermodynamic data of such complex species were primarily derived from macroscopic approaches, such as potentiometric and extraction experiments. ${ }^{6}$ However, the assignment of species to distinct molecular complexes with empirical formulas was mainly accomplished without structural information. In fact, the existence of most of the postulated species and their contributions to the multifaceted aqueous chemistry of the $\mathrm{UO}_{2}{ }^{2+}$ ion still await unequivocal evidence from spectroscopic or theoretical approaches. In recent years, progress in the advancement not only of spectroscopic techniques but also of quantum chemical (QC) approaches has provided an increasing amount of spectral data related to the aqueous speciation of $\mathrm{U}(\mathrm{VI})$. However, the state of knowledge still appears partially contradictory and unclear. ${ }^{9-12}$

The idea of a multimethodological round-robin test (RRT) on the aqueous speciation of an actinide system was born in the aftermath of the closing round table discussion at the first Workshop on Advanced Techniques in Actinide Spectroscopy (ATAS) held in November 2012 at Dresden, Germany. During this discussion, scientists working in experimental or theoretical disciplines of actinide research shared their perspectives on the contributions from the other discipline and their expectations associated therewith. It became obvious that the current knowledge is inconsistent because of the multitudinous data provided by various spectroscopic and theoretical approaches, the meaning and interpretability of which are often hard to convey to the highly specialized scientists in different disciplines. Hence, this interdisciplinary test on a relatively simple actinide complex system was intended to elucidate the collection of knowledge from data acquisition to the resulting interpretational approaches. Consistencies and discrepancies in the results and in the way of best practice obtained by each single spectroscopic or theoretical technique applied in various laboratories and their sources should be disclosed to elaborate the potentials and limitations of each single technique. Furthermore, the benefit of multimethodical approaches for the molecular study of a selected complex system was to be assessed (cf. Figure S1).

The selection of the chemical system to be investigated and of the analytical techniques to be applied by the participants was mainly based on experience within the actinide geochemistry community as well as the feasibility of performing experiments encompassing the broad array of techniques available in leading laboratories in the field of geochemical sciences. After extensive discussions between potential participants, the aqueous $\mathrm{U}(\mathrm{VI})$ acetate system appeared to be the most implementable system in terms of experimental effort, radiation safety, sample preparation, and applicability to sophisticated spectroscopic techniques, namely, vibrational, luminescence, X-ray absorption, and nuclear magnetic resonance (NMR) spectroscopy. For several reasons (e.g., organizational aspects, few number of participants, etc.), techniques, such as UV-vis absorption spectroscopy or mass spectrometry that may also provide high information value on species distribution, were not considered.

The U(VI) acetate system has been examined since the 1950s. Mainly thermodynamic data on the complexing species and recently structural information by the application of spectroscopy and quantum chemistry have been derived. Up to now, the presence of four species for this complexing system is discussed in the acidic $\mathrm{pH}$ range $(\mathrm{pH} \leq 4): \mathrm{UO}_{2}\left(\mathrm{H}_{2} \mathrm{O}\right)_{5}{ }^{2+}$, $\mathrm{UO}_{2} \mathrm{CH}_{3} \mathrm{COO}^{+}, \mathrm{UO}_{2}\left(\mathrm{CH}_{3} \mathrm{COO}\right)_{2}$, and $\mathrm{UO}_{2}\left(\mathrm{CH}_{3} \mathrm{COO}\right)_{3}{ }^{-}$, denoted below as the $(1,0),(1,1),(1,2)$, and $(1,3)$ species, respectively (cf. Figure S2). ${ }^{13}$ A more recent study takes fewer species into account. ${ }^{14}$ Because of the formation of insoluble hydrolyzed U(VI) solid phases, for example, schoepite, the $\mathrm{pH}$ range considered in this RRT was restricted to a maximum value of 3.5. The details of the RRT organization are provided in the following section and in the Supporting Information (Sections S1-S4). 
More than 40 scientists from 20 institutions joined the RRT. According to the technique applied, the results were initially collected by nominated scientists acting as cluster speakers for a single technique applied in several laboratories. During a special session of the second ATAS workshop held in November 2014, preliminary summary reports were presented and discussed extensively. This work represents the outcome of this RRT. First, a survey of the experimental and theoretical data obtained is given for each technique. Second, the results are combined into a discussion focusing on the consequences for the knowledge on the U(VI) acetate speciation. Finally, general conclusions and perspectives of the RRT are provided. We believe that the methodology as well as the outcome of this RRT appeals to a broad community of inorganic chemists, actinide chemists, analytical chemists, geochemists, and environmental engineers.

\section{ORGANIZATION OF THE RRT}

The RRT was initiated in late 2013 at the Helmholtz-Zentrum Dresden-Rossendorf (HZDR). After the approval of the participating institutions, five clusters were formed according to the spectroscopic and theoretical techniques, namely, vibrational spectroscopy (VIB, five institutions), laser-induced luminescence spectroscopy (LUM, nine institutions), NMR spectroscopy (five institutions), X-ray absorption spectroscopy (XAS, three laboratories located at three different European synchrotron sources), and QC calculations (QCC, six institutions) (cf. Figure S1). An overview of the clusters and the respective participating institutions is given in Table S1. For each cluster, a representative speaker was nominated.

For each analytical method, participants received four samples, denoted $\mathrm{U} 1-\mathrm{U} 4$, and respective reference samples, denoted B1-B4 (see Table S2). Those samples were uniformly prepared at HZDR. The participants received detailed information on sample preparation as it is given in the Supporting Information (Section S3). Apart from the requirement of measuring the samples as received, no further restrictions or guidelines were given to the participants to avoid significant impact on the specific approaches of the participants. The performance of the measurements and analysis of the raw data (e.g., data transformations, background subtraction, smoothing, deconvolution, etc.), as well as data interpretation, were entirely at the responsibility of each single operator or operating team. The Supporting Information (Section S4) provides detailed information on each cluster, describes experimental procedures, and lists all relevant setting parameters of the participants for each analytical method and quantum chemistry.

The period for active participation was from January to August 2014. Each participant submitted the raw data obtained, a detailed description of settings, the data analysis procedures applied, and the interpretational approaches of the derived molecular structures to the respective cluster speaker who in turn provided a summary report of the cluster to the organizers of the RRT.

\section{RESULTS AND DISCUSSION}

3.1. Results from the Clusters. All clusters delivered substantial data sets for intercomparison to determine consistencies and discrepancies within the applied analytical methods. For the sake of brevity, only a summary of the most important aspects can be given here. But, a description and interpretation of the whole data and its discussion with up-todate literature as well as of different interpretative approaches of the participants are given in detail in the Supporting Information (Section S5). Furthermore, the original data from each participant are given in Section S6.

3.1.1. Vibrational Spectroscopy. All vibrational spectra of the samples U1-U4 obtained by IR and Raman spectroscopy are shown in Figure 1. The focus is set on the spectral region of the asymmetric $\left(\nu_{3}\left(\mathrm{UO}_{2}\right)\right)$ and symmetric $\left(\nu_{1}\left(\mathrm{UO}_{2}\right)\right)$ stretching modes for clarity (Figure 1A,B). All other features observed in the spectra of the different samples mainly reflect the modes of the acetate molecule, which do not show a change in frequency but in intensity (Figures S9 and S10). The spectra provided by the single contributors are given in different colors. A detailed description of the spectroscopic features and their interpretation is given in the Supporting Information (Section S5.1).

The vibrational spectroscopic data of this RRT provided by five independent participants are in excellent agreement for all samples (cf. Table S8). For IR spectroscopy, the interpretation of IR data by all participants is consistent, and four different species could be spectrally identified. Generally, all species found in the IR spectra should be intrinsically observed in the

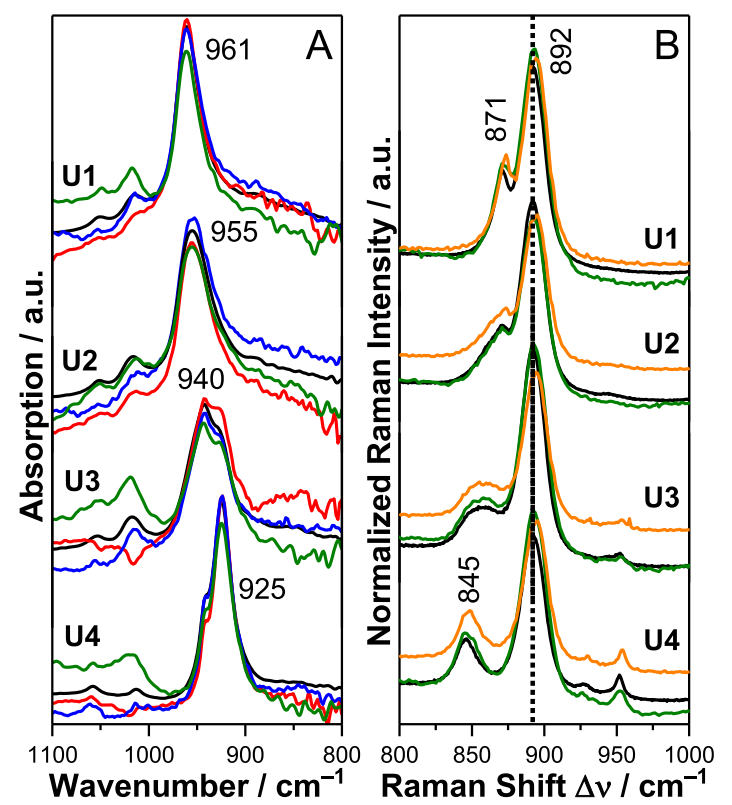

Figure 1. Vibrational spectra of samples U1-U4. Different colors correspond to different participants. Only the spectral regions of the $\nu_{3}\left(\mathrm{UO}_{2}\right)$ mode from IR $(\mathrm{A})$ and of the $\nu_{1}\left(\mathrm{UO}_{2}\right)$ mode from Raman spectroscopy (B) are shown. Values are given in $\mathrm{cm}^{-1}$.

Raman spectra as well. However, Raman spectra of the U(VI) acetate complexes are more difficult to interpret because of the strongly interfering bands of the symmetric stretching mode $\nu_{1}\left(\mathrm{UO}_{2}\right)$ of the different species and a vibrational mode of the acetate ligand. Consequently, the results of this cluster demonstrate that IR and Raman spectroscopy are strongly complementary techniques for the aqueous speciation of actinyl complexes.

3.1.2. Luminescence Spectroscopy. A survey of the timeresolved emission spectra revealed significantly different sets of acquisition parameters, resulting in a large time gate that ranged from a few nanoseconds to $100 \mathrm{~ms}$ (Figure 2A). Beside the gate width, parameters such as the initial time delay and the number and size of the gate steps strongly varied between the data sets 
provided by the participants (cf. Table S5 in the Supporting Information). However, general analysis of the time-resolved spectra from each participant suggested multi-exponential decay behavior, indicating the presence of at least two distinct species showing characteristic peak positions and luminescence lifetimes regardless of the variation in the spectral parameter applied (cf. Figures S11-S19 in the Supporting Information).

For a detailed analysis of the spectral data, the reader is referred to the Supporting Information. In summary, the strongly varying acquisition parameters applied by the participants of the LUM cluster required additional global data analysis prior to an adequate data evaluation (Figure 2B,C).
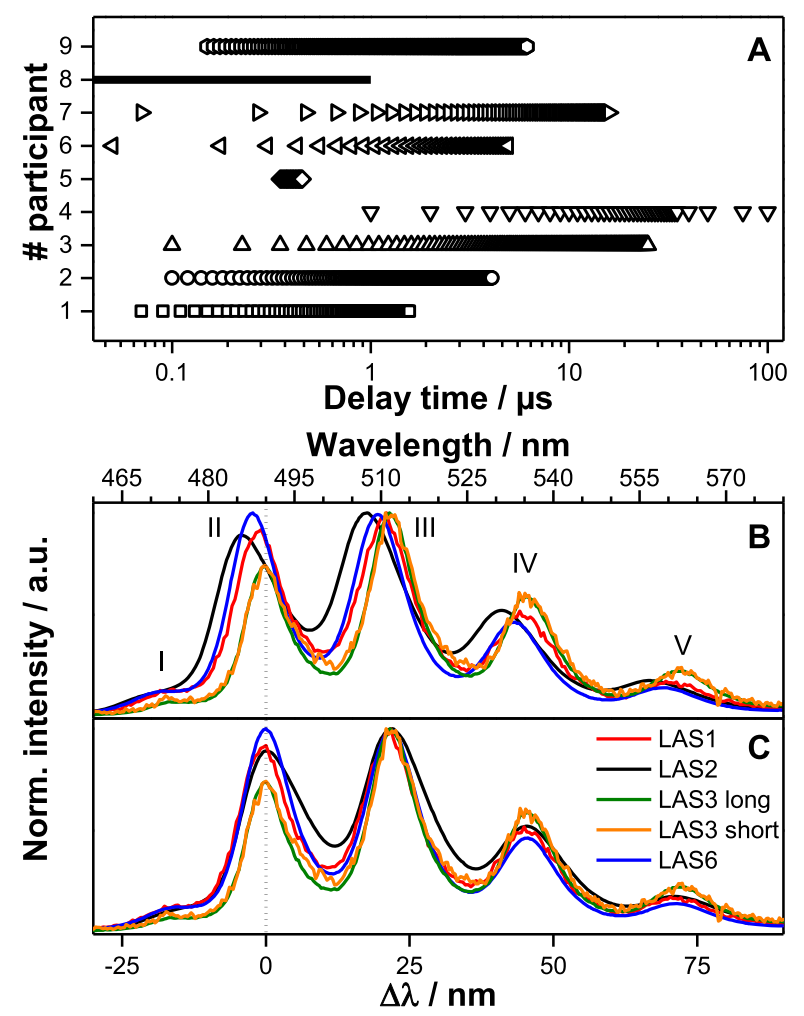

Figure 2. Time regimes applied by all participants of the LUM cluster during the acquisition of the luminescence spectra (A). Emission spectra of sample U1 as measured (B) and after calibration (C) using the peak maximum at $490 \mathrm{~nm}$ as an internal standard. Only spectra of four participants could be used for calibration (for details, see Supporting Information 5.2).

After an internal referencing of selected spectra, the relative peak positions show only marginal deviations, demonstrating the applicability of luminescence spectroscopy to the investigation of U(VI) speciation and the identification of single species. However, among the results of all participants, the obtained luminescence lifetimes displayed a broad distribution range. These discrepancies likely arose from the use of significantly differing experimental setups and algorithms for the spectral analysis. These aspects were already addressed during the first interlaboratory RRT on aqueous solutions of U(VI) studied by LUM. $^{15}$

3.1.3. X-ray Absorption Spectroscopy. The discussion of the XAS results will be focused on samples $\mathrm{U} 1(\mathrm{pH} 1)$ and $\mathrm{U} 4(\mathrm{pH}$ 3.5), which were analyzed by all groups (cf. Figure S20). As demonstrated in a recent comprehensive study, samples U2 ( $\mathrm{pH}$ 2 ) and $\mathrm{U} 3(\mathrm{pH} 2.5$ ) are known to contain species mixtures that are impossible to be unequivocally resolved by XAFS analysis based on four data points, that is, U(VI) acetate under four different $\mathrm{pH}$ conditions. ${ }^{13}$ A detailed discussion of the curvefitting results and their interpretation is given in the Supporting Information (Section S5.3). To sum up most important findings (Table 1), we can conclude that

- The data sets for samples U1 and U4 obtained at different beamlines are consistent.

- U1: the presence of the $(1,0)$ species was unambiguously confirmed by all three teams, and the structure reported in the literature was reproduced better than within typical extended X-ray absorption fine structure (EXAFS) error margins.

- U2 and U3: complex mixtures of all the species considered cannot be differentiated using EXAFS through measurements of only four data points, but a clear spectral trend is discernible from the EXAFS measurements of participants XAS-2 and -3 .

- U4: the dominant presence of the $(1,3)$ species was confirmed by all the cluster's participants. However, uncertainties in $\mathrm{CN}$ determination might cause different interpretations of the coordination schemes for the same data set.

The final point underpins the general importance of a priori speciation information that might be necessary for obtaining a conclusive picture from EXAFS curve fit analysis. Conversely, any EXAFS shell fitting analysis will be prone to be biased by such a priori knowledge.

3.1.4. NMR Spectroscopy. The NMR data are remarkably consistent and show little variation among the five independent groups. A detailed discussion of both ${ }^{1} \mathrm{H}$ and ${ }^{13} \mathrm{C}$ spectra is given in the Supporting Information (Section S5.4). The use of an internal standard as well as the reliability of modern NMR spectrometers allows excellent and reproducible results. Based on the control samples, the impact of the $\mathrm{pH}$ on the acetate spectra was found to be negligible. Hence, the speciation of the acetate molecule does not significantly change in this $\mathrm{pH}$ range. Conversely, systematic changes are observed in the spectra of the $\mathrm{U}(\mathrm{VI})$ acetate samples with increasing $\mathrm{pH}$. This effect is most dramatic in the ${ }^{13} \mathrm{C}$ spectra (Figure 3 ), where the signal representing the carboxyl group broadens considerably with $\mathrm{pH}$. This line broadening is attributed to the exchange between free and bound acetate groups, which is in the range of the relaxation time of the resonance signal. NMR spectroscopy can be used to examine high exchange rates of such molecular complexes in solution and has successfully been applied to binary and ternary $\mathrm{U}(\mathrm{VI})$ complexes. ${ }^{16}$ The observation of only one averaged peak per position in the ${ }^{1} \mathrm{H}$ and ${ }^{13} \mathrm{C}$ spectra of the $\mathrm{U}(\mathrm{VI})$ acetate complex system even at the higher field clearly indicates a fast exchange rate. The differences observed in the line widths of the signals measured at the same field (at $400 \mathrm{MHz}$ ) are mainly due to the variations in the homogeneity of the magnetic field, that is, due to the differences in the shimming procedure. At higher fields, the chemical shifts differ more (in $\mathrm{Hz}$ ) between the exchanging sites (free acetate and complexes), and the exchange-averaged signals are broader compared to those in the $400 \mathrm{MHz}$ spectra.

As neither the chemical shifts of the individual exchanging species nor their relative population are known, simulation of the spectra via a matrix formalism cannot be applied to extract kinetic information. Adequate results can be achieved only by running experiments at lower temperatures, but these are far 
Table 1. EXAFS Shell-Fitting Parameters ${ }^{a, h}$

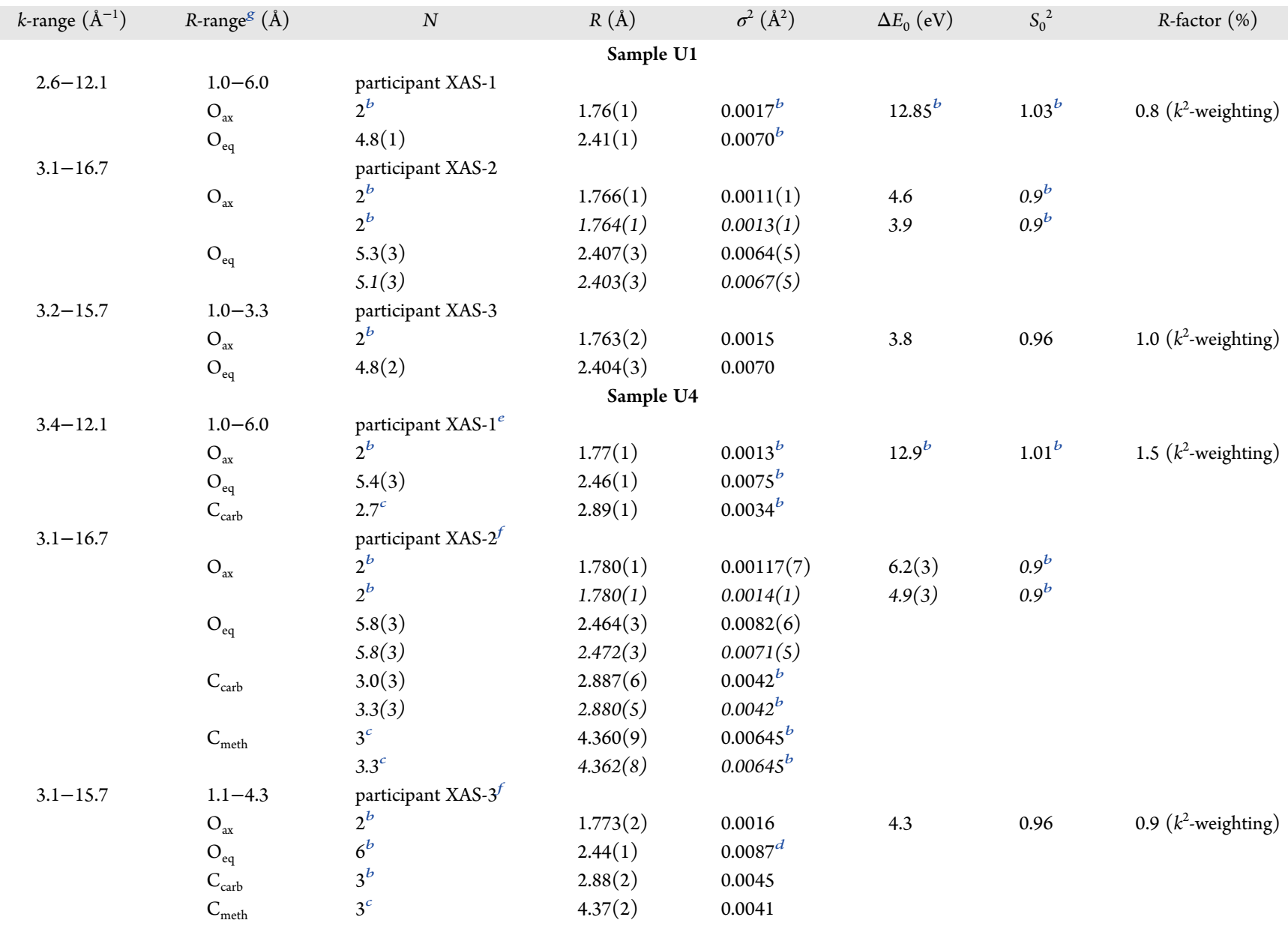

${ }^{a}$ The multiple scattering path $\mathrm{U}-\mathrm{O}_{\mathrm{ax} 1}-\mathrm{O}_{\mathrm{ax2}}-\mathrm{U}$ was included in all fits of sample $\mathrm{U} 1$, structural model: uranyl acetate dihydrate. ${ }^{20}{ }^{b}$ Fixed fit parameter. ${ }^{c}$ Parameter correlated with the preceding shell. ${ }^{d}$ Significant improvement by including asymmetry parameter (3rd cumulant). ${ }^{e} 3$ and 4 legs multiple scattering path $\mathrm{O}_{\mathrm{ax} 1}-\mathrm{U}-\mathrm{O}_{\mathrm{ax2}} .{ }^{f}$ Multiple scattering path $\mathrm{U}-\mathrm{O}_{\mathrm{ax1}}-\mathrm{O}_{\mathrm{ax} 2}-\mathrm{U}$ and $\mathrm{MS}$ paths to $\mathrm{C}_{\text {meth }}$ included, distances and DebyeWaller factors correlated with single scattering values. ${ }^{g}$ Values without phase shift correction. ${ }^{h}$ Values in italics given for participant XAS-2 represent literature data taken from ref 13 .

beyond the scope of this RRT. Nevertheless, the observations of increased linewidths and chemical shifts in the current data set are consistent with earlier findings, suggesting a correlation of the linewidths with the concentration of the $\mathrm{U}(\mathrm{VI})$ acetate species. $^{16}$

3.1.5. Theory/QCC. DFT is the most widely used method for calculations of uranyl(VI) complexes because U(VI) formally exhibits a $5 \mathrm{f}^{0}$ electronic configuration, and thus a singleconfigurational approach is appropriate. The effect of the spinorbit coupling is only marginal for most of the properties considered here, and therefore, it was neglected. We tested various DFT functionals and a wave function method (MP2). The structural and spectroscopic properties of the two most relevant species, that is, the complexes $(1,0)$ and $(1,3)$, and the energy of ligand replacement, that is from $(1,0)$ to $(1,3)$, were calculated. The results are given in Tables S13 and S14 (Supporting Information). The detailed discussion in the Supporting Information (Section S5.5) focuses on the results obtained with the same basis set for various methods (Programs Gaussian, TURBOMOLE, and ParaGauss), as summarized in Tables S10 and 2, together with ADF results in solution for comparison.
The results suggest that the calculated spectroscopic parameters depend considerably on the optimized structures. Generally, all methods satisfactorily reproduce the $\mathrm{U}-\mathrm{O}_{\mathrm{ax}}$ distances, whereas the $\mathrm{U}-\mathrm{O}_{\mathrm{eq}}$ distances are generally overestimated compared to experimental data. To fully evaluate the calculated results, they must be compared with the experimental results, which will be done in the next section.

3.2. Molecular Aspects of the U(VI) Speciation Derived from Each Cluster's Results. In this section, the results and interpretational approaches obtained from each cluster are analyzed with respect to the aqueous $\mathrm{U}(\mathrm{VI})$ acetate speciation. As a synopsis, Table 3 displays the consistency of the data obtained and the results of interpretational approaches. The predominant $\mathrm{U}(\mathrm{VI})$ species in samples $\mathrm{U} 1$ and $\mathrm{U} 4$ are expected to be the $(1,0)$ and $(1,3)$ species, respectively. At variance, for samples $\mathrm{U} 2$ and $\mathrm{U} 3$, the presence of several species is expected where the predominance of uranyl complexes with an increasing number of ligands increases with $\mathrm{pH}$. Thus, to evaluate the data across all clusters, the $(1,0)$ and $(1,3)$ species are discussed first.

The unequivocal data provided by the spectroscopic clusters for sample U1 facilitate the assignment of the spectral features observed to the predominant aqueous $(1,0)$ species. This conclusion is explicitly supported by the VIB and XAS clusters. 


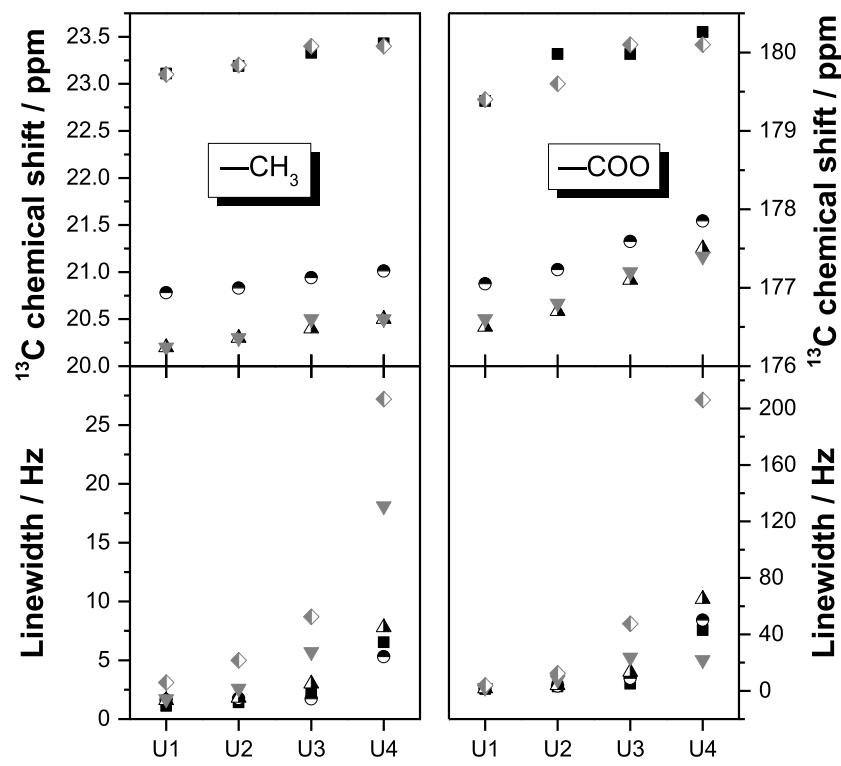

Figure $3 .{ }^{13} \mathrm{C}$ NMR spectroscopic data of the methyl (left) and carboxyl regions (right). Comparison of the chemical shifts (top) and linewidths (below) for samples U1-U4 obtained by different RRT participants. Note that for better comparison the same line broadening factor (lb) was applied to the raw data (see Tables S11 and S12).

All participants performing Raman and infrared spectroscopy concordantly assigned the spectral data showing the symmetric $\left(\nu_{1}\left(\mathrm{UO}_{2}\right)\right)$ and asymmetric $\left(\nu_{3}\left(\mathrm{UO}_{2}\right)\right)$ stretching vibrational modes to the fully hydrated uranyl ion. Furthermore, the structure of the aqueous uranyl complex, in which the $\mathrm{UO}_{2}{ }^{2+}$ moiety is coordinated by 5 water molecules in the equatorial plane, is unambiguously reproduced by EXAFS shell fitting by all three participating beamline groups. However, regarding the results of the U1 sample from the LUM cluster, the assignment of the predominant species as the $(1,0)$ species is mainly based on the prevailing conditions.

The $(1,3)$ species is unequivocally identified by all participants in the VIB and XAS clusters. Based on the highly consistent spectral data, the interpretational approaches used by the participants differ only slightly. Most participants interpreted the spectral data in terms of the predominance of the $(1,3)$ species with a structure, in which all three acetate ligands are bidentately coordinated to the uranyl moiety. From the VIB data, only one set of Raman spectra was not assigned to the $(1,3)$ species, while in one set of IR spectra, the presence of an even higher coordinated $\mathrm{U}(\mathrm{VI})$ acetate complex, the $(1,4)$ species, was discussed but was subsequently considered to be unlikely because of steric considerations. Moreover, according to one set of XAS data, the $(1,3)$ species was considered to exhibit a mixed type of coordination of the ligands, that is, two bidentately and one monodentately bound ligand.

In contrast, the assignment of spectral features to the $(1,1)$ and $(1,2)$ species is generally less clear. Only the vibrational spectra exhibit a spectral feature, namely the frequency of the $\nu\left(\mathrm{UO}_{2}\right)$ modes, that directly correlates with the formation of each species and thus allows the unambiguous assignment of four distinct species. However, even the interpretation of vibrational data is hampered by overlapping signals, and the assignment of these species is not completely consistent in case of the Raman spectra. Note, however, that the Raman spectroscopy results of this RRT convincingly revise previous literature data. ${ }^{17}$ For the XAS and LUM clusters, identifying these species is not possible for various reasons. For EXAFS spectroscopy, this is mainly due to the number of samples being too small, as shown previously. ${ }^{13}$ Moreover, it has been

Table 2. Average Aqueous Phase $\mathrm{U}-\mathrm{O}$ and $\mathrm{U}-\mathrm{C}_{\text {carb }}$ Bond Distances $(\AA), \mathrm{O}=\mathrm{U}=\mathrm{O} \nu_{1}$ and $\nu_{3}$ Stretching Vibrational Frequencies $\left(\mathrm{cm}^{-1}\right)$, and NMR Chemical Shifts $(\mathrm{ppm})$ of the $(1,0)$ and $(1,3)$ Species as Well as Gibbs Energy of Formation of $\mathrm{UO}_{2}\left(\mathrm{CH}_{3} \mathrm{COO}\right)_{3}{ }^{-} \Delta G\left(\mathrm{kcal} \mathrm{mol}^{-1}\right)$ at Different Theoretical Levels ${ }^{a}$

\begin{tabular}{|c|c|c|c|c|c|c|c|c|c|c|c|c|c|c|c|c|}
\hline & \multirow[b]{3}{*}{$b$} & \multicolumn{5}{|c|}{$\mathrm{UO}_{2}\left(\mathrm{H}_{2} \mathrm{O}\right)_{5}{ }^{2+}$} & \multicolumn{9}{|c|}{$\mathrm{UO}_{2}\left(\mathrm{CH}_{3} \mathrm{COO}\right)_{3}{ }^{-}$} & \multirow{3}{*}{$\frac{\text { energy }}{\Delta G^{d}}$} \\
\hline & & \multicolumn{2}{|c|}{ bond distances } & \multicolumn{3}{|c|}{ vibr. frequencies } & \multicolumn{3}{|c|}{ bond distances } & \multicolumn{3}{|c|}{ vibr. frequencies } & \multicolumn{3}{|c|}{ NMR chemical shift ${ }^{c}$} & \\
\hline & & $\mathrm{U}-\mathrm{O}_{\mathrm{ax}}$ & $\mathrm{U}-\mathrm{O}_{\mathrm{eq}}$ & $\bar{\nu}$ & $\nu_{3}$ & $\nu_{3}-\nu_{1}$ & $\mathrm{U}-\mathrm{O}_{\mathrm{ax}}$ & $\mathrm{U}-\mathrm{O}_{\mathrm{eq}}$ & $\overline{\mathrm{U}-\mathrm{C}_{\text {carb }}}$ & $\nu_{1}$ & $\nu_{3}$ & $\nu_{3}-\nu_{1}$ & ${ }^{1} \mathrm{HCH}_{3}$ & ${ }^{13} \mathrm{CCH}_{3}$ & ${ }^{13} \mathrm{CCOO}$ & \\
\hline MP2 & G & 1.767 & 2.420 & 896 & 988 & 92 & 1.796 & 2.450 & 2.832 & 833 & 922 & 99 & & & & \\
\hline \multirow[t]{2}{*}{ BP86 } & G & 1.774 & 2.467 & 880 & 951 & 71 & 1.811 & 2.492 & 2.879 & 799 & 863 & 64 & 2.3 & 25.9 & 194.1 & -99.0 \\
\hline & $\mathrm{P}$ & 1.780 & 2.444 & 857 & 923 & 66 & 1.809 & 2.482 & 2.874 & 795 & 852 & 57 & & & & -85.7 \\
\hline \multirow[t]{2}{*}{ PBE } & $\mathrm{P}$ & 1.777 & 2.443 & 863 & 929 & 66 & 1.806 & 2.482 & 2.872 & 801 & 857 & 56 & & & & -83.8 \\
\hline & A & 1.779 & 2.453 & 865 & 921 & 56 & 1.818 & 2.482 & 2.877 & 784 & 829 & 45 & 2.5 & 26.0 & 208.3 & -92.3 \\
\hline \multirow[t]{3}{*}{ PBE0 } & G & 1.734 & 2.454 & 967 & 1026 & 59 & 1.765 & 2.471 & 2.853 & 894 & 949 & 55 & 2.5 & 24.8 & 197.7 & -94.5 \\
\hline & $P$ & 1.739 & 2.431 & 956 & 1004 & 48 & 1.764 & 2.464 & 2.851 & 890 & 935 & 45 & & & & -82.3 \\
\hline & A & 1.742 & 2.444 & 953 & 991 & 38 & 1.775 & 2.465 & 2.852 & 869 & 901 & 32 & 2.6 & 26.7 & 206.6 & -87.6 \\
\hline \multirow[t]{2}{*}{ B3LYP } & G & 1.752 & 2.474 & 930 & 994 & 64 & 1.785 & 2.497 & 2.884 & 856 & 915 & 59 & 2.4 & 25.3 & 200.4 & -92.3 \\
\hline & A & 1.762 & 2.460 & 912 & 954 & 42 & 1.797 & 2.490 & 2.883 & 832 & 866 & 34 & 2.6 & 27.1 & 209.8 & -86.4 \\
\hline \multirow[t]{3}{*}{ M06L } & G & 1.747 & 2.477 & 926 & 995 & 69 & 1.782 & 2.496 & 2.877 & 840 & 906 & 66 & 2.4 & 24.8 & 188.1 & -97.1 \\
\hline & $\mathrm{P}$ & 1.752 & 2.458 & 902 & 962 & 60 & 1.781 & 2.490 & 2.875 & 836 & 891 & 55 & & & & -90.7 \\
\hline & A & 1.750 & 2.464 & 916 & 958 & 42 & 1.790 & 2.486 & 2.871 & 816 & 855 & 39 & & & & -92.1 \\
\hline \multirow[t]{2}{*}{ M06 } & G & 1.728 & 2.470 & 980 & 1034 & 54 & 1.756 & 2.497 & 2.876 & 916 & 968 & 52 & 2.4 & 23.8 & 190.7 & -86.6 \\
\hline & $\mathrm{P}$ & 1.733 & 2.451 & 983 & 1026 & 43 & 1.755 & 2.490 & 2.874 & 909 & 950 & 41 & & & & -81.0 \\
\hline Exp. $^{e}$ & & 1.763 & 2.406 & 871 & 961 & 90 & 1.774 & 2.456 & 2.885 & 846 & 923 & 77 & 2.1 & 21.9 & 178.8 & \\
\hline
\end{tabular}

${ }^{a}$ Corresponding experimental values are given in the bottom row. ${ }^{b} \mathrm{G}$ : Gaussian 09 and P: ParaGauss utilizing segmented new small core ECP on $\mathrm{U}^{21}$ and cc-pvtz on the rest except for MP2 calculations in which $6-311+\mathrm{G}^{*}$ basis set was used for all elements except U. A: ADF utilizing ZORATZ2P basis set without frozen core approximation. ${ }^{c}$ Calculated NMR chemical shifts are relative to TMS; in the experiments, DSS was used. ${ }^{d}$ For the reaction $\mathrm{UO}_{2}\left(\mathrm{H}_{2} \mathrm{O}\right)_{5}{ }^{2+}+3 \mathrm{CH}_{3} \mathrm{COO}^{-} \rightarrow \mathrm{UO}_{2}\left(\mathrm{CH}_{3} \mathrm{COO}\right)_{3}{ }^{-}+5 \mathrm{H}_{2} \mathrm{O}$. ${ }^{e}$ Experimental values: distances from Table 1, averaged. Vibrational frequencies from Table S8, averaged. NMR parameters from Table S11, averaged. 
Table 3. Qualitative Summary of the Clusters' Outcome with Respect to the U(VI) Acetate Species ${ }^{a}$

\begin{tabular}{lcccc} 
& \multicolumn{4}{c}{$\mathrm{U}(\mathrm{VI})$ species } \\
\cline { 2 - 5 } cluster & $(1,0) \mathrm{UO}_{2}\left(\mathrm{H}_{2} \mathrm{O}\right)_{5}{ }^{2+}$ & $(1,1) \mathrm{UO}_{2}\left(\mathrm{CH}_{3} \mathrm{COO}\right)^{+}$ & $(1,2) \mathrm{UO}_{2}\left(\mathrm{CH}_{3} \mathrm{COO}\right)_{2}$ & $(1,3) \mathrm{UO}_{2}\left(\mathrm{CH}_{3} \mathrm{COO}_{3}{ }^{-}\right.$ \\
VIB & $+/+$ & $+/+$ & $+/+(\mathrm{IR})$ & $+/+(\mathrm{IR})$ \\
& & & $+/-(\mathrm{Raman})^{b}$ & $+/-(\mathrm{Raman})^{b}$ \\
XAS & $+/+$ & $+/-^{c}$ & $+/-^{c}$ & $+/-^{d}$ \\
NMR & $+/+$ & & & $+/+$ \\
LUM & $-/-^{e}$ & & & \\
QCC & $+/-^{f}$ & N/A & N/A
\end{tabular}

$a_{+} /+$: high consistency of data and interpretation between the participants; $+/-$: high consistency of data and ambiguous interpretation between the participants; -/-: low consistency of data and interpretation if available; N/A: not available. ${ }^{b}$ Deviation in assignment \& interpretation. ${ }^{c}$ Unequivocal identification not feasible. ${ }^{d}$ Deviation in interpretation of structure. ${ }^{e}$ Due to low sample number nearly no interpretation approaches; global equilibration procedure required. ${ }^{f}$ Variation due to weakly bound $\mathrm{H}_{2} \mathrm{O}$-ligands.

demonstrated that the spectrum of the $(1,2)$ species intrinsically cannot be derived from EXAFS data. ${ }^{13}$ Indeed, the LUM data from all contributors demonstrate that each sample shows its characteristic spectral fingerprint; however, specific species can be extracted only when an extensive parametric analysis of a larger data set is available than the one generated within this RRT.

NMR spectroscopy is a non-optical spectroscopic technique that exclusively provides signals reflecting changes of the energy differences in nuclear spin states in hydrogen and carbon atoms of the ligand, which differentiates this technique from the other spectroscopic methods used in this RRT. On the one hand, the slight shifts observed in the ${ }^{1} \mathrm{H}$ and ${ }^{13} \mathrm{C}$ spectra nicely represent the ongoing complexation of the uranyl moiety by acetate molecules as the $\mathrm{pH}$ increases. On the other hand, an assignment of the signals observed to distinct species is not possible. As already stated in Section 3.1.4, a more detailed analysis yielding speciation information can be obtained only by conducting much more extensive experiments, which are beyond the scope of this RRT.

A principal concern of this RRT was the juxtaposition of the results obtained from the experimental and theoretical approaches. For the sake of simplicity, the calculations focused on the $(1,0)$ and $(1,3)$ species assuming the penta-aquo complex $\mathrm{UO}_{2}\left(\mathrm{H}_{2} \mathrm{O}\right)_{5}{ }^{2+}$ for the $(1,0)$ species and the exclusively bidentate complex $\mathrm{UO}_{2}\left(\mathrm{CH}_{3} \mathrm{COO}\right)_{3}{ }^{-}$for the $(1,3)$ species.

In general, the accuracy of the calculated values of the interatomic distances and the vibrational frequencies agree well with experimental results but the quality of the results depends slightly on the method applied (Table 2). Compared to the structures in the gas phase (Table S10), the calculations including solvation effects provide slightly longer $\mathrm{U}-\mathrm{O}_{\mathrm{ax}}$ distances and slightly shorter $\mathrm{U}-\mathrm{O}_{\text {eq }}$ distances (Tables S10 and 2). Solvation effects slightly improve the agreement with EXAFS results for $\mathrm{U}-\mathrm{O}_{\mathrm{eq}}$ and in some cases also for $\mathrm{U}-\mathrm{O}_{\mathrm{ax}}$ distances. As an example, the solvation calculations of both the $(1,0)$ and $(1,3)$ species at the MP2 level gave bond distances that are all within $0.02 \AA$ of the values from the EXAFS data (Table $2)$. At the DFT level, the deviations are slightly larger. Depending on the type of functional, the deviations from the experimental bond distances can be as large as $0.1 \AA$ (Table S14). For all methods, a trend to overestimating the lengths of $\mathrm{U}-\mathrm{O}$ bonds to the ligands has to be noted, by $0.06 \AA$ for $(1,0)$ and by $0.03 \AA$ for $(1,3)$ on average. The geometry differences between the species $(1,0)$ and $(1,3)$ are qualitatively reproduced by all methods. The increase of $\mathrm{U}-\mathrm{O}_{\mathrm{ax}}$ by about $0.01 \AA$ und the increase of $\mathrm{U}-\mathrm{O}_{\text {eq }}$ by about $0.05 \AA$ due to complexation, as observed by EXAFS, are overestimated for $\mathrm{U}-\mathrm{O}_{\mathrm{ax}}$ and underestimated for $\mathrm{U}-\mathrm{O}_{\text {eq }}$ by about $0.02 \AA$ in all calculations (Table 2). In general, calculated differences of properties should be more accurate than absolute values due to favorable error cancelation. On the other hand, the observed deviations from experiment are not unexpected due to limitations of the solvation modeling in the calculations and due to some contributions from species with less than three acetate ligands in probe U4 in the experiment (see Figure S2).

The calculated harmonic vibrational frequencies of the $\nu_{1}$ and $\nu_{3}\left(\mathrm{UO}_{2}\right)$ modes are consistent with the experimental values. Not surprisingly, the better the agreement between the experimental and theoretical bond distances, the better tends to be the agreement in vibrational frequencies (irrespective of gas phase or aqueous phase calculations). For instance, the calculations at the MP2 level, which coincide well with the experimental bond distances, provide an average deviation of only $17 \mathrm{~cm}^{-1}$ (max. 27 $\mathrm{cm}^{-1}$ ) between the theoretical and experimental $\nu_{1} / \nu_{3}\left(\mathrm{UO}_{2}\right)$ frequencies (Table 2). The results obtained with the M06L functional with ParaGauss are only slightly less accurate. In line with the overestimation of the increase of the $\mathrm{U}-\mathrm{O}_{\mathrm{ax}}$ bond due to acetate complexation in the calculations, the red shift of the uranyl stretching vibrations as a result of acetate complexation, which amounts to about $30 \mathrm{~cm}^{-1}$ in experiment, is overestimated by all methods by at least $30 \mathrm{~cm}^{-1}$ (Table 2). The difference between $\nu_{3}$ and $\nu_{1}$ is well reproduced by MP2 (Table 2, overestimated for $(1,0)=2 \mathrm{~cm}^{-1}$, for $\left.(1,3)=13 \mathrm{~cm}^{-1}\right)$, but underestimated by DFT by $40 \mathrm{~cm}^{-1}$ for $(1,0)$ and $30 \mathrm{~cm}^{-1}$ for $(1,3)$ on average (Table 2$)$. These findings of systematic deviations support the assumption of differences between experiment and theory due to model aspects in the calculations and additional species in the experimental probes (see above).

Besides, it should be noted that there are three essential sources of errors in computations: the model choice, the electronic structure method, including the particular (relativistic) treatment of the core electrons, and the solvation treatment. Commonly some error cancelation may be expected. One may therefore always argue that good agreement with experiments may simply be a coincidence and computational results always warrant a critical evaluation.

This RRT demonstrates that the basic molecular parameters of small actinide complexes obtained using QCC are accurate with respect to the experimental findings. In particular, the interatomic distances are obviously predicted with higher accuracy than vibrational frequencies (for which rescaling is a rather common practice). Deviations of calculated parameters from the experimental values, averaged over all methods applied, amount to $1-2.5 \%$ for bond lengths and to $4-6 \%$ for the uranyl stretching vibrations. For well-defined systems and with a proper 
choice of the method, an even better agreement with experiment is achievable. Small systematic deviations, especially of differences of properties, point to limitations of the models used in the calculations and uncertainties of speciation in the experimental probes: thus, such differences are of special interest and are helpful for improving calculations as well as the interpretation of experiments.

\section{CONCLUSIONS AND PERSPECTIVES}

4.1. Evaluation of the RRT. The concept of this RRT based on the contributions of a broad array of spectroscopic techniques and $\mathrm{QC}$ approaches to the aqueous $\mathrm{U}(\mathrm{VI})$ speciation exemplified for the U(VI) acetate system. This project intended to collect results from as many laboratories with dedicated expertise in the molecular analysis of actinides as possible. Hence, for the sake of practicability, the scale of the RRT had to be restricted to a relatively small number of samples, which can hardly be considered as adequate for a thorough investigation of an aqueous speciation as complex as the U(VI) acetate system. In consequence, a conclusive evaluation of the RRT shall be restricted to some general, yet still-instructive aspects. Table 4 summarizes the major findings of the applied techniques as well as their strengths and limitations concerning the U-acetate complex system.

The generally high consistency of the data, obtained within each cluster, reflects the accuracy and suitability of the techniques applied for the exploration of the uranyl complex system. In particular, those techniques conducted on commercial instruments provided highly consistent data, as shown by the results of the VIB and NMR clusters. Despite having worked on different beamlines and individually adapted setups, even accelerator-driven XAS provided highly consistent raw data. Regarding the characteristic specifications of the single facilities, one of the merits of this RRT is explicitly demonstrating the reproducibility of the performance of the three participating synchrotron beamlines.

A distinct comparison of the luminescence spectral data was only feasible after performing a correction routine for wavelength calibration, as described in Sections 3.1.2 and S5.2. At first, the consistency of luminescence data appeared to be quite low. This inconsistency might raise questions about the comparability of the spectral analysis, which was performed based on only simple spectral fingerprinting published previously. Conversely, when the spectra were referenced internally to the spectral distances of the peaks, the data within the LUM cluster demonstrated high reproducibility. However, considering the data of the luminescence decay curves provided by the respective participants, a comparison of the spectral data is hardly possible, as described in Section S5.2. In fact, these aspects were already addressed in a previous RRT in $2003 .{ }^{15}$

Multimethodological approaches to the analysis of molecular complexes are expected to benefit from the acquisition of complementary information. Potentially, this advantage might be derived from IR and Raman spectroscopy because of the intrinsic selection rules of the vibrational modes observed in the respective spectra. In fact, four prevailing species and the interacting functional groups in the molecular complexes could be evidently derived from the vibrational spectra (see Sections 3.1.1 and S5.1). This information was nicely reflected by the results in the NMR cluster, which provided evidence for the increasing degree of complexation of the uranyl moiety with increasing $\mathrm{pH}$ (see Sections 3.1.4 and S5.4). As the results from the LUM and XAS cluster indicated the presence of only three

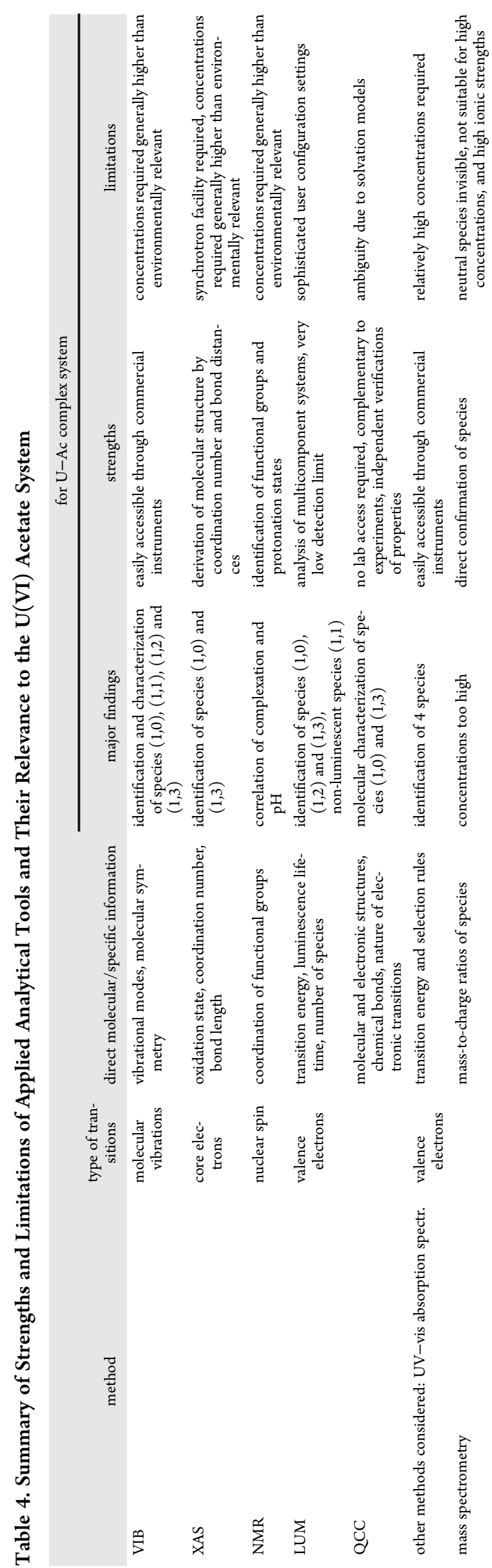


species, the missing complementarity of these techniques with the former ones must be discussed in more detail.

Due to the intrinsic properties of the EXAFS spectra, the $(1,2)$ species cannot be differentiated from the other species which is largely due to symmetry reasons as already mentioned above (see Sections 3.1.3 and S5.3). Hence, such intrinsic limitations, which might not be obvious even to advanced spectroscopists, must be understood prior to performing extensive series of experiments. The intrinsic luminescence properties of the species might also be responsible for the lower number of species observed using LUM. One of the species-most likely the $(1,1)$ species-was assumed to be not luminescent. Although an ultimate verification is still lacking, this explanation would clearly elucidate why comprehensive complementary information was not obtained from the LUM cluster. However, it has to be emphasized that the number of samples was certainly insufficient for a thorough investigation of the speciation, particularly in the case of luminescent spectroscopy.

Consequently, to gain maximum benefit from a multimethodological approach exploring the U(VI) acetate speciation, the techniques must be carefully selected, which requires a detailed knowledge of their capabilities and restrictions. Even then, experienced users might suffer from unexplored details of intrinsic properties of the molecules under investigation. At this point, theoretical calculations, as they represent an independent and thus complementary approach to molecular properties, appear particularly valuable. As shown in this RRT, results from various $\mathrm{QC}$ codes are very consistent when the same stationary points of the species under study are considered. Bond distances are well reproduced with the MP2 and a number of DFT approaches, while vibrational frequencies show slightly larger deviations, but are still useful for the assignment of the spectral findings to distinct molecular structures. Nevertheless, the general trends of geometry and vibrational parameters of the $\mathrm{U}(\mathrm{VI})$ acetate system are calculated in agreement with the experiment. Small systematic deviations from the experimental results, which are independent of the detailed method applied, are of special interest, as they point to weaknesses of the models applied and/or the need for a more detailed understanding of the system considered in the experiments. DFT calculations predict chemical shifts of NMR signals quite precisely. In the future, the development of algorithms providing reliable calculations of luminescent spectra is desired.

In summary, this RRT was very successful in terms of the high number of participants throughout the community and their acceptance to share their expertise and experimental (unpublished) data. Furthermore, the cooperation and willingness of the participants to contribute to the discussions during the compilation process of the RRT illustrate the profound interest in improving the performance of the various techniques and in continuing the knowledge transfer among scientists from various analytical disciplines to obtain a deeper understanding of basic molecular complexes in actinide chemistry.

4.2. Scope and Perspectives. In the past decades, significant progress in laser technology, microfluidics, digital signal processing, and the development of novel light detectors has contributed to dramatic efficiency enhancements of spectroscopic methods in terms of significantly reduced accumulation times and increase in quality of spectra. Consequently, due to improved signal-to-noise ratios in the spectra, the extraction of further molecular information by numerical analysis has gained importance in spectroscopic investigations. Apart from classical analysis methods, such as curve decomposition or factor analysis, which have been applied intensively ever since, various statistical methods-chemometrics in particular-have been developed for processing these data and extracting essential information. However, only the accurate application of such statistical methods prohibits from misinterpretation of the results, which might lead to erroneous understanding otherwise. ${ }^{18}$ This particularly refers to wellestablished fitting procedures, which often appear to be poorly documented in scientific articles. Hence, the provision of comprehensive numerical information necessary for the reproduction of such an analysis is indispensable. Moreover, we felt confident through this RRT that it is necessary to establish a method utilizing chemometrics as a routine tool for characterization of molecular structures. In the future, further development of dimension reduction and feature extraction methods such as self-organizing maps is foreseen to contribute further to molecular spectroscopy.

A key concern of this RRT was the elicitation of the mutual promotion of QC calculations and spectroscopic approaches. The results presented provide a widely consistent molecular understanding of the $(1,0)$ and $(1,3)$ uranyl acetate species, even though theoretical predictions and experimental findings showed some discrepancies to a different extent with respect to the different spectroscopic techniques. Hence, the development or improvement of algorithms explicitly considering the intrinsic properties of specific spectroscopic techniques is expected to enhance the concordance of theoretical and experimental approaches and appears therefore necessary. If an intense communicative exchange of theoreticians and experimentalists generates a mutual understanding of their operational modes and mindsets, future work might proceed in extracting specific information from complex experimental data and vice versa in verifying theoretical investigations by experimental findings.

On a technical level of instrumentation, this RRT demonstrated the importance of the availability of experimental parameters. Only detailed sets of relevant instrumental parameters strengthen the sustainability of the data presented and might provide the basis for future technical improvements. In this context, it was shown that a commonly accepted and applied calibration routine for LUM is mandatory though hard to be implemented throughout worldwide researchers. Nevertheless, we suggest a suitable calibration approach in the Supporting Information (cf. Figure S21). This approach follows the procedure already described earlier using the emission line of a conventional $\mathrm{Hg}$ lamp. ${ }^{19}$ In the future, it is eligible to represent luminescence spectra on an energy scale (wavenumber units) instead of a wavelength scale. This would facilitate the comparability of spectral features observed under different experimental setups, thereby data interpretation by various researchers becoming more perspicuous and comprehensible.

\section{ASSOCIATED CONTENT}

\section{S Supporting Information}

The Supporting Information is available free of charge on the ACS Publications website at DOI: 10.1021/acsomega.9b00164.

Further information on the general RRT organization; $\mathrm{U}(\mathrm{VI})$ acetate calculated speciation; experimental details on sample preparation; clusters and experimental parameters of the spectroscopic setups including VIB, LUM, XAS, and NMR spectroscopy and QCC programs, methods, and basis sets with detailed description and 
interpretation of the results from each cluster; presentation of all data provided by the participants; calibration routine for LUM; and extended list of references (PDF)

\section{AUTHOR INFORMATION}

\section{Corresponding Author}

*E-mail: k.mueller@hzdr.de.

\section{ORCID}

Katharina Müller: 0000-0002-0038-1638

Harald Foerstendorf: 0000-0002-8334-9317

Satoru Tsushima: 0000-0002-4520-6147

Michael U. Kumke: 0000-0002-3395-9379

Grégory Lefêvre: 0000-0002-1054-906X

Harris Mason: 0000-0002-1840-0550

Ping Yang: 0000-0003-4726-2860

Christian K. R. Adam: 0000-0003-1449-7065

Herman M. Cho: 0000-0001-7072-2306

Christophe Den Auwer: 0000-0003-2880-0280

Björn Drobot: 0000-0003-1245-0466

Peter Kaden: 0000-0002-9414-2936

Alena Kremleva: 0000-0003-3330-0862

James A. Platts: 0000-0002-1008-6595

Robert Polly: 0000-0002-7024-7987

Brian A. Powell: 0000-0003-0423-0180

Notker Rösch: 0000-0002-4769-4332

Andreas C. Scheinost: 0000-0002-6608-5428

Georg Schreckenbach: 0000-0002-4614-0901

Andrej Skerencak-Frech: 0000-0003-2177-4462

Vladimir Sladkov: 0000-0002-1053-0473

\section{Notes}

The authors declare no competing financial interest.

\section{ACKNOWLEDGMENTS}

We would like to thank Annika Maffert, Christa Müller, and Heidrun Neubert for sample preparation and packaging and Annette Rumpel, Ines Prescher, and Christine Roch for their assistance with radiation safety and shipment procedures. For analytical support, we acknowledge Karsten Heim, Sophia Kostudis, and Michael Steppert. Furthermore, we kindly acknowledge the proofreading and the fruitful discussions with Vinzenz Brendler, Clemens Walther, Astrid Barkleit, Atsushi Ikeda-Ohno, Michael Patzschke, and Jochen Autschbach. We appreciate the synchrotron light sources SOLEIL at Gif-surYvette, France, ESRF at Grenoble, France, and KIT Synchrotron Light Source at Karlsruhe, Germany, for the provision of beamtime. B.D. and K.B were supported by the German Federal Ministry of Economic Affairs and Energy (BMWi) under contract numbers 02E11415G and 02E11011. P.Y. was supported for this work by the Heavy Element Chemistry program at LANL sponsored by the United States Department of Energy Office of Basic Energy Sciences. Los Alamos National Laboratory is operated by Los Alamos National Security, LLC, for the National Nuclear Security Administration of the United States Department of Energy under Contract DE-AC5206NA25396. G.S. acknowledges funding from the Natural Sciences and Engineering Council of Canada (NSERC, Discovery Grant). B.A.P. Experimental was supported by the U.S. Department of Energy, Office of Science, Office of Basic Energy Sciences, under Award Number DE-SC0010355. Work at LLNL was supported by the Subsurface Biogeochemical
Research Program of the U.S. Department of Energy's Office of Biological and Environmental Research. Prepared by LLNL under contract DE-AC52-07NA27344. The work of I.C., A.K. and S.K. has been supported by the German Federal Ministry of Economic Affairs and Energy (BMWi) under contract nos. 02E11001 and 02E11415E. This material is based upon work supported by the U.S. Department of Energy Office of Science, Office of Basic Energy Sciences, Division of Chemical Sciences, Geosciences and Biosciences, Heavy Element Chemistry program, and was performed in the William R. Wiley Environmental Molecular Sciences Laboratory, a D.O.E. national scientific user facility sponsored by the Department of Energy's Office of Biological and Environmental Research and located at the Pacific Northwest National Laboratory (PNNL). PNNL is a multiprogram national laboratory operated for D.O.E. by Battelle.

\section{REFERENCES}

(1) Choppin, G. R. Actinide speciation in the environment. J. Radioanal. Nucl. Chem. 2007, 273, 695-703.

(2) Maher, K.; Bargar, J. R.; Brown, G. E. Environmental speciation of actinides. Inorg. Chem. 2013, 52, 3510-3532.

(3) Geckeis, H.; Lützenkirchen, J.; Polly, R.; Rabung, T.; Schmidt, M. Mineral-water interface reactions of actinides. Chem. Rev. 2013, 113, $1016-1062$

(4) Burns, P. C.; Ewing, R. C.; Navrotsky, A. Nuclear fuel in a reactor accident. Science 2012, 335, 1184-1188.

(5) Berto, S.; Crea, F.; Daniele, P. G.; Gianguzza, A.; Pettignano, A.; Sammartano, S. Advances in the investigation of dioxouranium(VI) complexes of interest for natural fluids. Coord. Chem. Rev. 2012, 256, $63-81$.

(6) Guillaumont, R.; Fanghänel, T.; Fuger, J.; Grenthe, I.; Neck, V.; Palmer, D. A.; Rand, M. H. Update on the Chemical Thermodynamics of U, Np, Pu, Am and Tc; Elsevier: Amsterdam, 2003; p 970.

(7) Hummel, W.; Anderegg, G.; Rao, L.; Puigdomènech, I.; Tochiyama, O. Chemical Thermodynamics of Compounds and Complexes of $\mathrm{U}, \mathrm{Np}, \mathrm{Pu}, \mathrm{Am}, \mathrm{Tc}, \mathrm{Se}, \mathrm{Ni}$ and $\mathrm{Zr}$ with Selected Organic Ligands; Elsevier: Amsterdam, 2005; Vol. 9, p 1132.

(8) Campbell, K. M.; Gallegos, T. J.; Landa, E. R. Biogeochemical aspects of uranium mineralization, mining, milling, and remediation. Appl. Geochem. 2015, 57, 206-235.

(9) Drobot, B.; Bauer, A.; Steudtner, R.; Tsushima, S.; Bok, F.; Patzschke, M.; Raff, J.; Brendler, V. Speciation studies of metals in trace concentrations: the mononuclear uranyl(VI) hydroxo complexes. Anal. Chem. 2016, 88, 3548-3555.

(10) Müller, K.; Brendler, V.; Foerstendorf, H. Aqueous uranium(VI) hydrolysis species characterized by attenuated total reflection fouriertransform infrared spectroscopy. Inorg. Chem. 2008, 47, 10127-10134.

(11) Moll, H.; Rossberg, A.; Steudtner, R.; Drobot, B.; Müller, K.; Tsushima, S. Uranium(VI) chemistry in strong alkaline solution: speciation and oxygen exchange mechanism. Inorg. Chem. 2014, 53, $1585-1593$.

(12) Drobot, B.; Steudtner, R.; Raff, J.; Geipel, G.; Brendler, V.; Tsushima, S. Combining luminescence spectroscopy, parallel factor analysis and quantum chemistry to reveal metal speciation - a case study of uranyl(VI) hydrolysis. Chem. Sci. 2015, 6, 964-972.

(13) Lucks, C.; Rossberg, A.; Tsushima, S.; Foerstendorf, H.; Scheinost, A. C.; Bernhard, G. Aqueous uranium(VI) complexes with acetic and succinic acid: speciation and structure revisited. Inorg. Chem. 2012, 51, 12288-12300.

(14) Sladkov, V. Photochemical characterization of uranyl interaction with acetic acid. J. Photochem. Photobiol., A 2014, 295, 40-45.

(15) Billard, I.; Ansoborlo, E.; Apperson, K.; Arpigny, S.; Azenha, M. E.; Birch, D.; Bros, P.; Burrows, H. D.; Choppin, G.; Couston, L.; Dubois, V.; Fanghänel, T.; Geipel, G.; Hubert, S.; Kim, J. I.; Kimura, T.; Klenze, R.; Kronenberg, A.; Kumke, M.; Lagarde, G.; Lamarque, G.; Lis, S.; Madic, C.; Meinrath, G.; Moulin, C.; Nagaishi, R.; Parker, D.; 
Plancque, G.; Scherbaum, F.; Simoni, E.; Sinkov, S.; Viallesoubranne, C. Aqueous solutions of uranium(VI) as studied by time-resolved emission spectroscopy: A round-robin test. Appl. Spectrosc. 2003, 57, 1027-1038.

(16) Aas, W.; Szabó, Z.; Grenthe, I. Equilibria and dynamics in binary and ternary uranyl oxalate and acetate/fluoride complexes. J. Chem. Soc., Dalton Trans. 1999, 1311-1318.

(17) Quilès, F.; Burneau, A. Infrared and Raman spectroscopic study of uranyl complexes: hydroxide and acetate derivatives in aqueous solution. Vib. Spectrosc. 1998, 18, 61-75.

(18) Shashilov, V. A.; Lednev, I. K. Advanced Statistical and Numerical Methods for Spectroscopic Characterization of Protein Structural Evolution. Chem. Rev. 2010, 110, 5692-5713.

(19) Martinsen, P.; Jordan, B.; McGlone, A.; Gaastra, P.; Laurie, T. Accurate and precise wavelength calibration for wide bandwidth array spectrometers. Appl. Spectrosc. 2008, 62, 1008-1012.

(20) Howatson, J.; Grev, D. M.; Morosin, B. Crystal and molecular structure of uranyl acetate dihydrate. J. Inorg. Nucl. Chem. 1975, 37, $1933-1935$

(21) Küchle, W.; Dolg, M.; Stoll, H.; Preuss, H. Energy-adjusted pseudopotentials for the actinides. Parameter sets and test calculations for thorium and thorium monoxide. J. Chem. Phys. 1994, 100, 75357542. 